\title{
The Exact Law of Large Numbers for Independent Random Matching*
}

\author{
Darrell Duffie ${ }^{\dagger}$ and Yeneng Sun ${ }^{\ddagger}$
}

August 31, 2004

\begin{abstract}
This paper provides micro-foundations for independent random matching of a large population, as widely used in the economics literature. We consider both static and dynamic systems with random mutation, partial matching arising from search, and type changes induced by matching. Under independence assumptions at each randomization step, we show that there is an almost-sure constant cross-sectional distribution of types in a large population, and moreover that the multi-period cross-sectional distribution of types is deterministic and evolves according to the transition matrices of the type process of a given agent.
\end{abstract}

*This work was initiated in July 2000 while the second author visited Stanford University. We are grateful for comments from Pierre-Olivier Weill and Zhixiang Zhang.

${ }^{\dagger}$ Graduate School of Business, Stanford University, Stanford, CA 94305-5015. e-mail: duffie@stanford.edu

${ }^{\ddagger}$ Department of Mathematics, National University of Singapore, 2 Science Drive 2, Singapore 117543; and Department of Economics, National University of Singapore, 1 Arts Link, Singapore 117570. e-mail: matsuny@nus.edu.sg 


\section{Introduction}

We provide an exact law of large numbers for independent random matching, under which there is an almost-sure constant cross-sectional distribution of types in a large population. We address both static and dynamic systems with random mutation, partial matching arising from search, and type changes induced by matching. Based on a suitable measure-theoretic framework, an exact law of large numbers is proved for each case under an independence assumption on each of the randomization steps: matching, mutation, and matching-induced type changes. The time evolution of the cross-sectional distribution of types is completely determined by the agent-level Markov chain for type, with explicitly calculated transition matrices.

A deterministic (almost surely) cross-sectional distribution of types in independent random matching models for continuum populations had been widely used in several literatures, without a foundation. Economists and geneticists, among others, have implicitly or explicitly assumed the law of large numbers for independent random matching in a continuum population, by which we mean a non-atomic measure space of agents. This result is relied upon in large literatures within general equilibrium theory (e.g. [21], [22], [39], [52]), game theory (e.g. [4], [6], [9], [20], [27]), monetary theory (e.g. [12], [25], [28], [32], [33], [44], [49]), labor economics (e.g. [11], [29], [41], [42], [43]), illiquid financial markets (e.g. [15], [16], [34], [50], [51]), and biology (e.g. [7], [26], [38]). Mathematical foundations, however, have been lacking, as has been noted by Green and Zhou [25].

We provide the exact law of large numbers for random pairwise matching ${ }^{1}$ by formulating a suitable independence condition in types. ${ }^{2}$ In a companion paper [17], we prove the existence of a joint agent-probability space satisfying the independence conditions that we require here.

For a simple illustration, suppose that each agent within a fraction $p$ of a continuum population has an item for sale, and that the agents in the remaining fraction $q=1-p$ are in need of the item. If the agents "pair off independently," a notion that we formalize shortly, then each would-be seller meets some would-be buyer with probability $q$. At such a meeting, a trade occurs. One presumes that, almost surely, in a natural model, exactly a fraction $q$ of the seller population would trade, implying that a fraction $q p$ of the total population are sellers

\footnotetext{
${ }^{1}$ Various versions of the exact law of large numbers for a continuum of random variables (also for a continuum of stochastic processes or correspondences) have been provided in [45]-[48]. These results consider the average, or the distribution (or finite-dimensional distributions) of a given family of random variables (or stochastic processes).

${ }^{2}$ The independence condition we propose is natural, but may not be obvious. For example, a random matching in a finite population may not allow independence among agents since the matching of agent $i$ to agent $j$ implies of course that $j$ is also matched to $i$, implying some correlation among agents. The effect of this correlation is reduced to zero in a continuum population. A new concept, "Markov conditional independence in types," is proposed for dynamic matching, under which the transition law at each randomization step depends on only the previous one or two steps of randomization.
} 
who trade, that the same fraction $p q$ of the total population are buyers who trade, and that the fraction of the population that would not trade is $1-2 p q$. Among other results, we show that this presumption is correct in a suitable mathematical framework. In [17], moreover, we prove that such a model exists.

Hellwig [28] is the first, to our knowledge, to have relied on the effect of the exact law of large numbers for random pairwise matching in a market, in a study of a monetary exchange economy. ${ }^{3}$ Much earlier reliance can be found in genetics. In 1908, G.H. Hardy [26] and W. Weinberg (see [7]) independently proposed that with random mating in a large population, one could determine the constant fractions of each allele in the population. Hardy wrote: "suppose that the numbers are fairly large, so that the mating may be regarded as random," and then used, in effect, an exact law of large numbers for random matching to deduce his results. ${ }^{4}$ For a simple illustration, consider a continuum population of gametes consisting of two alleles, $A$ and $B$, in initial proportions $p$ and $q=1-p$. Then, following the Hardy-Weinberg approach, the new population would have a fraction $p^{2}$ whose parents are both of type $A$, a fraction $q^{2}$ whose parents are both of type $B$, and a fraction $2 p q$ whose parents are of mixed type (heterozygotes). These genotypic proportions asserted by Hardy and Weinberg are already, implicitly, based on an exact law of large numbers for random matching in a large population. In order to consider the implications for the steady-state distribution of alleles, suppose that, with both parents of allele $A$, the offspring are of allele $A$, and with both parents of allele $B$, the offspring are of allele $B$. Suppose that the offspring of parents of different alleles are, say, equally likely to be of allele $A$ or allele $B$. The Hardy-Weinberg equilibrium for this special case is a population with steady-state constant proportions $p=60 \%$ of allele $A$ and $q=40 \%$ of allele $B$. Provided that the law of large numbers for random matching indeed applies, this is verified by checking that, if generation $k$ has this cross-sectional distribution, then the fraction of allele $A$ in generation $k+1$ is almost surely $0.6^{2}+0.5 \times(2 \times 0.6 \times 0.4)=0.6$. This Hardy-Weinberg Law, governing steady-state allelic and genotypic frequencies, is a special case of our results treating dynamics and steady-state behavior.

In applications, random-matching models have also allowed for random mutation of agents, obviously in genetics, and in economics via random changes in preferences, productivity, or endowments. Typical models are also based on "random search," meaning that the time at

\footnotetext{
${ }^{3}$ Diamond [10] had earlier treated random matching of a large population with, in effect, finitely many employers, but not pairwise matching within a large population. The matching of a large population with a finite population can be treated directly by the exact law of large numbers for a continuum of independent random variables. For example, let $N(i)$ be the event that worker $i$ is matched with an employer of a given type, and suppose this event is pairwise independent and of the same probability $p$, in a continuum population of such workers. Then, under the conditions of [48], the fraction of the population that is matched to this type of employer is $p$, almost surely.

${ }^{4}$ Later in his article, Hardy did go on to consider the effect of "casual deviations," and the issue of stability.
} 
which a given agent is matched is also uncertain. With random search, during each given time period, some fraction of the agents are not matched. Finally, in some cases, it is important that the impact of a match between two agents on their post-match types is itself random, as in [33] and [42]. For instance, trade sometimes depends on a favorable outcome of a productivity shock to the buyer, allowing the buyer to produce, or not, the output necessary to pay the seller. In some models, once paired by matching, agents use mixed strategies for their actions, causing another stage of random type changes. It is also often the case that one wishes not only an (almost surely) deterministic cross-sectional distribution of types as a result of each round of matching, but also a cross-sectional distribution of types that is constant over time, as in the Hardy-Weinberg Equilibrium. It may also help if one knows the finite dimensional time distributions of the cross-sectional type process almost surely. We provide a collection of results treating all of these cases.

Our results include the potential for random birth and death, because we allow for random mutation of types, which can include "alive" or "dead." We do not, however, consider population growth (or declines), which could be handled by relatively straightforward extensions of the results here, that we leave for future work. It would also be straightforward to extend our results in order to consider the effect of "aggregate shocks," for example common adjustments to the parameters determining speed of matching, according to a Markov chain, as in the business-cycle effects on employer-worker matching studied by ${ }^{5}$ Mortensen and Pissarides [42]. When we treat dynamic models, we take only the discrete-time case, although continuous-time models of random matching are also popular (e.g. [15], [42], [49], [51]). We are in the process of extending our results to the continuous-time setting, which requires different methods.

We consider three basic issues. The first is a rigorous formulation of independent random matching for a continuum population in a meaningful theoretical framework. The second is a proof of the exact law of large numbers for a continuum population with general independent random matchings. This involves the development of general results suitable for other applications involving independent random matching. The third issue is the existence of independent random matchings in the general framework, which is reported here, but studied systematically in a companion paper [17].

Since there are fundamental measurability problems associated with a continuum of independent random variables, ${ }^{6}$ there has, up to now, been no theoretical treatment of the exact law of large numbers for independent random matching among a continuum population.

\footnotetext{
${ }^{5}$ Ljungqvist and Sargent [35] present a discrete-time version of the Mortensen-Pissarides model, which is further treated in discrete time by Cole and Rogerson [8] and by Merz [40].

${ }^{6}$ See, for example, [2], [13], [14], [18], [30] and discussions in [45] and [48].
} 
In [48], various versions of the exact law of large numbers and their converses are proved by direct application of simple measure-theoretic methods in the framework of an extension of the usual product probability space that retains the Fubini property. ${ }^{7}$ This paper adopts the measure-theoretic framework of [48].

The remainder of the paper is organized as follows. Section 2 is a user's guide, going immediately to the form and implications of the main results, and putting off most of the necessary mathematical developments. In Section 3, we consider random full and partial matchings in the static case after a brief introduction of the measure-theoretic framework (a Fubini extension of the usual product probability space) in Section 3.1. A random full matching is formally defined in Section 3.2. A key point is to formulate the independence condition on the type processes of agents being matched, rather than on the matching function itself. ${ }^{8}$ This allows a simple application of the exact law of large numbers from [45] and [48], so as to obtain immediately an exact law of large numbers for an independent random full matching in Theorem 1. Random partial matchings (the case of search models) are considered in Section 3.3, where it is shown in Theorem 2 that, almost surely, a deterministic proportion of agents of each type are not matched, while the complementary portion are matched to other agents in proportion to their presence in population.

Section 4 considers a dynamical system for agent types, allowing for random mutation, partial matching, and match-induced random type changes. We introduce the condition of Markov conditional independence to model the idea that at every time period, (1) an independent random mutation follows from the previous period, (2) it is then followed by an independent random partial matching, (3) finally, there is independent random type changing for matched agents. Markov conditional independence allows us to show that the individual type processes of almost-all agents are essentially pairwise independent Markov chains. This leads to a demonstration that there is an almost-sure constant cross-sectional distribution of types in a large population (including stationarity of the cross-sectional distribution of agent types), and moreover, that the time evolution of the cross-sectional distribution of types is (almost surely) completely determined as that of a Markov chain with known transition matrices. All of these results are included in Theorem 3.

\footnotetext{
${ }^{7}$ It is easy to construct examples of a continuum of independent random variables whose sample means or distributions are constant (see, for example, [2] and [24] and [30]). However, the difficulty is that one can also construct other examples of a pathological process with a continuum of independent random variables whose sample functions may not be measurable, or behave in a very "strange" way (such as being equal to any given function on the continuum almost surely, as in [30] and [48]). By working with a Fubini extension of the usual product probability space, one is able to obtain general results on the exact law of large numbers, as in [48], without the possibility of constructing the type of pathological processes.

${ }^{8}$ We call this "independence in types," meaning that for essentially every pair $(i, j)$ of agents, the type of the agent to be randomly matched with agent $i$ is independent of the type of the agent to be randomly matched with agent $j$.
} 
Existence results for random matching, in static settings and in dynamic settings that are (Markov conditionally) independent in types, are stated without proofs in Section 5. These results are fully developed in a companion paper [17]. While the proofs for the exact law of large numbers for the independent random matchings use only simple probabilistic manipulations, the proofs of the existence results in [17] make extensive use of nonstandard analysis. ${ }^{9}$ The existence result for the dynamic setting is particularly intricate because one must construct a continuum of independent Markov chains that is derived from random mutation, random partial matching and random type changing.

A brief discussion of the relevant literature is given in Section 6. Proofs of Theorems 1, 2 and 3 are given in the appendix.

Finally, we emphasize again that we must work with extensions of the usual product measure spaces (of agents and states of the world), since a process formed by a continuum of independent random variables is never measurable with respect to the completion of the usual product $\sigma$-algebra, except in the trivial case that almost all the random variables in the process are constants. ${ }^{10}$

\section{User's Guide}

This section gives a simple understanding of some of the key results, without detailing most of the definitions and arguments that we later use to formalize and prove these results.

We fix a probability space $(\Omega, \mathcal{F}, P)$ representing uncertainty, an atomless probability space $(I, \mathcal{I}, \lambda)$ representing the set of agents, ${ }^{11}$ and a finite agent-type space $S=\{1, \ldots, K\}$. One may take the set $I$ of agents to be, for example, the unit interval [0,1] (or, as noted in [17], a set with cardinality of the continuum that can be constructed as an equivalence class of a sequence of finite sets).

In order to discuss independent random matching, we consider a product probability space $(I \times \Omega, \mathcal{W}, Q)$ such that $\mathcal{W}$ contains the product $\sigma$-algebra $\mathcal{I} \otimes \mathcal{F}$, and such that the marginals of $Q$ on $(I, \mathcal{I})$ and $(\Omega, \mathcal{F})$ are $\lambda$ and $P$ respectively. This extension $(I \times \Omega, \mathcal{W}, Q)$ of the product of the two underlying spaces must have the basic Fubini property in order for the following law-of-large numbers results to make sense.

A cross-sectional or probability distribution of types is an element of $\Delta=\left\{p \in \mathbb{R}_{+}^{K}\right.$ :

\footnotetext{
${ }^{9}$ Note that the existence results themselves are stated in Section 5 using common measure-theoretic terms. A reader interested only in applications of the exact law of large numbers for independent random matching could rely on this paper. The companion paper [17] is accessible to those with some knowledge of nonstandard analysis, which can be gained, for example, from the first three chapters of the book [37].

${ }^{10}$ See, for example, Proposition 1.1 in [46].

${ }^{11} \mathrm{~A}$ probability space $(I, \mathcal{I}, \lambda)$ is atomless if there does not exist $A \in \mathcal{I}$ such that $\lambda(A)>0$, and for any $\mathcal{I}$-measurable subset $C$ of $A, \lambda(C)=0$ or $\lambda(C)=\lambda(A)$.
} 
$\left.p_{1}+\cdots+p_{K}=1\right\}$.

For each non-negative integer time $n$, some $\mathcal{W}$-measurable $\alpha^{n}: I \times \Omega \rightarrow S$ specifies the current type $\alpha^{n}(i, \omega)$ of agent $i \in I$ in state of the world $\omega$. The associated cross-sectional distribution of types at time $n$ is the $\Delta$-valued random variable $p^{n}(\omega)$ (also denoted by $p_{\omega}^{n}$ ) defined by

$$
p_{k}^{n}(\omega)=\lambda\left(\left\{i \in I: \alpha^{n}(i, \omega)=k\right\}\right) .
$$

The initial type function $\alpha^{0}: I \rightarrow S$ is non-random.

For each time $n$, there is some random matching function $\pi^{n}: I \times \Omega \rightarrow I \cup\{J\}$, where $\{J\}$ is a singleton representing 'unmatched,' that specifies either an agent $j=\pi^{n}(i, \omega) \neq i$ in $I$ to whom $i$ is matched in state $\omega$, or specifies the outcome $\pi^{n}(i, \omega)=J$ that $i$ is not matched. It must be the case that if $i$ is matched to $j$, then $j$ is matched to $i$. Specifically, for all $\omega$, $i$, and $j, \pi^{n}(i, \omega)=j$ if and only if $\pi^{n}(j, \omega)=i$. Let $g^{n}$ be a $\mathcal{W}$-measurable matching type function on $I \times \Omega$ into $S \cup\{J\}$, such that $g^{n}(i, \omega)$ is the type of the agent $j=\pi^{n}(i, \omega)$ who is matched with agent $i$ in state of nature $\omega$, or $g^{n}(i, \omega)=J$ if $\pi^{n}(i, \omega)=J$. As usual, we let $g_{i}^{n}$ and $\alpha_{i}^{n}$ denote the random variables whose outcomes in state $\omega$ are $g^{n}(i, \omega)$ and $\alpha^{n}(i, \omega)$, respectively. There is also some $\mathcal{W}$-measurable random mutation function $h^{n}: I \times \Omega \rightarrow S$ that specifies a mutated type for agent $i$ at state of nature $\omega$. For a realized state of nature $\omega, h_{\omega}^{n}$, $\pi_{\omega}^{n}, g_{\omega}^{n}$ and $\alpha_{\omega}^{n}$ denote, respectively, the realized mutation, matching, matching type and type functions on $I$.

The parameters of a random matching model with type space $S$ are

1. Some initial cross-sectional distribution $p^{0} \in \Delta$ of types (the type distribution induced by $\left.\alpha^{0}\right)$.

2. A $K \times K$ transition matrix $b$ fixing the probability $b_{k l}$ that an agent of type $k$ mutates to an agent of type $l$ in a given period, before matching.

3. Some $q \in[0,1]^{S}$ specifying, for each type $k$, the probability $q_{k}$ that an agent of type $k$ is not matched within one period. An agent who is not matched keeps her type in a given period, but may mutate to another type at the beginning of next period.

4. Some $\nu: S \times S \rightarrow \Delta$ specifying the probability $\nu_{k l}(r)$ that an agent of type $k$ who is matched with an agent of type $l$ will become, after matching, an agent of type $r$.

Fixing the parameters $\left(p^{0}, b, q, \nu\right)$ of some random matching model, under a natural definition of "Markov conditional independence for mutation, matching, and type changing" which we provide later in this paper, one conjectures the following results. ${ }^{12}$

\footnotetext{
${ }^{12}$ Models with random full matching, or with deterministic match-induced type changing, or without random
} 
- At each time $n \geq 1$, the realized cross-sectional type distribution $p^{n}(\omega)$ is $P$-almost surely (henceforth, "a.s.") equal to the expected cross-sectional type distribution $\bar{p}^{n}=$ $\int_{\Omega} p^{n}(\omega) d P(\omega)$.

- After the random mutation step at time $n$, the fraction of the population of a given type $l$ is almost surely $\sum_{k=1}^{K} \bar{p}_{k}^{n-1} b_{k l}$, denoted by $\bar{p}_{l}^{n-1 / 2}$.

- At each time $n \geq 1$ and for any type $k$, the fraction of the population of type $k$ that are not matched ${ }^{13}$ at period $n$ is

$$
\lambda\left(\left\{i \in I: h_{\omega}^{n}(i)=k, g_{\omega}^{n}(i)=J\right\}\right)=\bar{p}_{k}^{n-1 / 2} q_{k} \quad \text { a.s. }
$$

For any types $k, l \in S$, the fraction of the population who are agents of type $k$ that are matched with agents of type $l$ is

$$
\lambda\left(\left\{i: h_{\omega}^{n}(i)=k, g_{\omega}^{n}(i)=l\right\}\right)=\frac{\bar{p}_{k}^{n-1 / 2}\left(1-q_{k}\right) \bar{p}_{l}^{n-1 / 2}\left(1-q_{l}\right)}{\sum_{r=1}^{K} \bar{p}_{r}^{n-1 / 2}\left(1-q_{r}\right)} \text { a.s. }
$$

- At the end of each time period $n \geq 1$ (after match-induced type changing), for each type $r$, the new fraction of agents of type $r$ is

$$
p_{r}^{n}(\omega)=\bar{p}_{r}^{n}=\bar{p}_{r}^{n-1 / 2} q_{r}+\sum_{k, l=1}^{K} \frac{\nu_{k l}(r) \bar{p}_{k}^{n-1 / 2}\left(1-q_{k}\right) \bar{p}_{l}^{n-1 / 2}\left(1-q_{l}\right)}{\sum_{t=1}^{K} \bar{p}_{t}^{n-1 / 2}\left(1-q_{t}\right)} \text { a.s. }
$$

Using the fact that $\bar{p}_{l}^{n-1 / 2}=\sum_{k=1}^{K} \bar{p}_{k}^{n-1} b_{k l}$, one has a recursive formula for $\bar{p}^{n}$ in terms of $\bar{p}^{n-1}$, and thus $\bar{p}^{n}$ (and also $p^{n}$ ) can be computed directly from $p^{0}$.

- For $\lambda$-almost every agent $i \in I$, the type process $\alpha_{i}^{0}, \alpha_{i}^{1}, \alpha_{i}^{2}, \ldots$ is an $S$-valued Markov chain, ${ }^{14}$ with a $K \times K$ transition matrix $z^{n}$ specifying the probability of transition from type $k$ at time $n-1$ to type $l$ at time $n$ (for $n \geq 1$ ), given by

$$
z_{k l}^{n}=P\left(\alpha_{i}^{n}=l \mid \alpha_{i}^{n-1}=k\right)=q_{l} b_{k l}+\sum_{r, t=1}^{K} \nu_{r t}(l) b_{k r} \frac{\left(1-q_{r}\right)\left(1-q_{t}\right) \bar{p}_{t}^{n-1 / 2}}{\sum_{r^{\prime}=1}^{K}\left(1-q_{r^{\prime}}\right) \bar{p}_{r^{\prime}}^{n-1 / 2}},
$$

provided the event $\left\{\alpha_{i}^{n-1}=k\right\}$ has positive probability.

mutation, are special cases of our model. To avoid random mutation, one can simply take $b_{k k}$ to be one for all $k \in S$. If $q_{k}=0$ for all $k \in S$, then an agent will be matched with probability one. For $k, l \in S$, if $\nu_{k l}(r)$ is one for some $r$, then the match-induced type change is deterministic.

${ }^{13}$ We note that $\sum_{r=1}^{K} \bar{p}_{r}^{n-1 / 2}\left(1-q_{r}\right)$ is the fraction of population who are matched, while $\left(\bar{p}_{l}^{n-1 / 2}\left(1-q_{l}\right)\right) /\left(\sum_{r=1}^{K} \bar{p}_{r}^{n-1 / 2}\left(1-q_{r}\right)\right)$ is the relative fraction of the population who are matched agents of type $l$ among all matched agents.

${ }^{14}$ For a complete statement of what constitutes a Markov process, one must fix a filtration $\left\{\mathcal{F}_{0}, \mathcal{F}_{1}, \ldots\right\}$ of sub- $\sigma$-algebras of $\mathcal{F}$. For our purposes, it is natural, and suffices for this result, to take $\mathcal{F}_{t}$ to be the $\sigma$-algebra generated by $\left\{\alpha_{i}^{s}: i \in I, s \leq t\right\}$. 
- For $P$-almost every state of nature $\omega \in \Omega$, the cross-sectional type process $\alpha^{0}, \alpha_{\omega}^{1}, \alpha_{\omega}^{2}, \ldots$ is an $S$-valued Markov chain with the transition matrix $z^{n}$ at time $n-1$ and initial type distribution $p^{0}$. Thus, the evolution of the fractions of each type is deterministic and coincides with the evolution of the probability distribution of type for a given agent, except for the initial distributions. ${ }^{15}$

Given the mutation, search and match-induced type chaning parameters $(b, q, \nu)$, one also conjectures that, under the assumption of "Markov conditional independence," there is some steady-state constant cross-sectional type distribution $p^{*}$ in $\Delta$, in the sense that, for the parameters $\left(p^{*}, b, q, \nu\right)$ we have, almost surely, for all $n \geq 0, p_{\omega}^{n}=p^{*}$. Moreover, the Markov chains for the type process $\alpha_{i}^{0}, \alpha_{i}^{1}, \alpha_{i}^{2}, \ldots$ (for $\lambda$-almost every agent $i \in I$ ) and for the cross-sectional type process $\alpha^{0}, \alpha_{\omega}^{1}, \alpha_{\omega}^{2}, \ldots$ (for $P$-almost every state of nature $\omega \in \Omega$ ) are time-homogeneous, and the latter has $p^{*}$ as a stationary distribution. That is, for some fixed transition matrix $z$, we have $z^{n}=z$ for all $n \geq 1$, and we have, for all $\ell$ in $S$,

$$
\sum_{k=1}^{K} z_{k l} p_{k}^{*}=p_{\ell}^{*}
$$

We will demonstrate all of the results stated above, based on the following version of the exact law of large numbers, proved in Sun [45] and [48]. Given some $\mathcal{W}$-measurable $f$ : $I \times \Omega \rightarrow X$, where $X$ is a finite set (we state the result for general $X$ in Section 3.1), the random variables $\left\{f_{i}: i \in I\right\}$, defined by $f_{i}(\omega)=f(i, \omega)$, are said to be essentially pairwise independent ${ }^{16}$ if for $\lambda$-almost all $i \in I$, the random variables $f_{i}$ and $f_{j}$ are independent for $\lambda$ almost all $j \in I$. For brevity, in this case we say that $f$ itself is essentially pairwise independent. With the assumption of the Fubini property on $(I \times \Omega, \mathcal{W}, Q)$, the exact law of large numbers in [45] and [48] (which is stated as Lemma 1 in Section 7.1, for the convenience of the reader) says that if $f$ is essentially pairwise independent, then the sample functions $f_{\omega}$ have essentially constant distributions. Then, the notion of Markov conditional independence is used to derive the essential pairwise independence of the $n$-th period mutation, matching and type processes $h^{n}, g^{n}$ and $\alpha^{n}$, as well as the essential pairwise independence of the Markov chains $\alpha_{i}^{0}, \alpha_{i}^{1}, \alpha_{i}^{2}, \ldots$, which imply all the results stated above.

\footnotetext{
${ }^{15}$ We do not take the initial probability distribution of agent $i$ 's type to be $p^{0}$, but rather the Dirac measure at the type $\alpha^{0}(i)$. See Footnote 25 for a generalization.

${ }^{16}$ This condition is weaker than pairwise independence since each agent is allowed to have correlation with a null set of agents (including finitely many agents since a finite set is null under an atomless measure). For example, the agent space $I$ is divided into a continuum of cohorts, with each cohort containing a fixed number $L$ of agents $(L \in \mathbb{N}$ ). If the agents across cohorts act independently (agents within each cohort may have correlation), then the essential pairwise independence condition is satisfied.
} 


\section{Exact law of large numbers for independent random matchings}

In this section, we consider independent random matchings, full or partial, in a static setting. Some background definitions are given in Section 3.1. Exact laws of large numbers for random full and partial matchings are presented, respectively, in Sections 3.2 and 3.3, and their proofs are given in the Appendix, Section 7.2.

\subsection{Some background definitions}

Let probability spaces $(I, \mathcal{I}, \lambda)$ and $(\Omega, \mathcal{F}, P)$ be our index and sample spaces respectively. ${ }^{17}$ In our applications, $(I, \mathcal{I}, \lambda)$ is an atomless probability space that is used to index the agents. If one prefers, $I$ can be taken to be the unit interval $[0,1]$. Let $(I \times \Omega, \mathcal{I} \otimes \mathcal{F}, \lambda \otimes P)$ be the usual product probability space. For a function $f$ on $I \times \Omega$ (not necessarily $\mathcal{I} \otimes \mathcal{F}$-measurable), and for $(i, \omega) \in I \times \Omega, f_{i}$ represents the function $f(i, \cdot)$ on $\Omega$, and $f_{\omega}$ the function $f(\cdot, \omega)$ on $I$.

In order to work with independent type processes arising from random matching, we need to work with an extension of the usual measure-theoretic product that retains the Fubini property. A formal definition, as in [48], is as follows.

Definition 1 A probability space $(I \times \Omega, \mathcal{W}, Q)$ extending the usual product space $(I \times \Omega, \mathcal{I} \otimes$ $\mathcal{F}, \lambda \otimes P)$ is said to be a Fubini extension of $(I \times \Omega, \mathcal{I} \otimes \mathcal{F}, \lambda \otimes P)$ if for any real-valued $Q$ integrable function $g$ on $(I \times \Omega, \mathcal{W})$, the functions $g_{i}=g(i, \cdot)$ and $g_{\omega}=f(\cdot, \omega)$ are integrable respectively on $(\Omega, \mathcal{F}, P)$ for $\lambda$-almost all $i \in i$ and on $(I, \mathcal{I}, \lambda)$ for P-almost all $\omega \in \Omega$; and if, moreover, $\int_{\Omega} g_{i} d P$ and $\int_{I} g_{\omega} d \lambda$ are integrable respectively on $(I, \mathcal{I}, \lambda)$ and on $(\Omega, \mathcal{F}, P)$, with $\int_{I \times \Omega} g d Q=\int_{I}\left(\int_{\Omega} g_{i} d P\right) d \lambda=\int_{\Omega}\left(\int_{I} g_{\omega} d \lambda\right) d P$. To reflect the fact that the probability space $(I \times \Omega, \mathcal{W}, Q)$ has $(I, \mathcal{I}, \lambda)$ and $(\Omega, \mathcal{F}, P)$ as its marginal spaces, as required by the Fubini property, it will be denoted by $(I \times \Omega, \mathcal{I} \otimes \mathcal{F}, \lambda \otimes P)$.

An $\mathcal{I} \otimes \mathcal{F}$-measurable function $f$ will also be called a process, each $f_{i}$ will be called a random variable of this process, and each $f_{\omega}$ will be called a sample function of the process.

We now introduce the following crucial independence condition. We state the definition using a complete separable metric space $X$ for the sake of generality; in particular, a finite space or an Euclidean space is a complete separable metric space.

Definition 2 An $\mathcal{I} \otimes \mathcal{F}$-measurable process $f$ from $I \times \Omega$ to a complete separable metric space $X$ is said to be essentially pairwise independent if for $\lambda$-almost all $i \in I$, the random variables $f_{i}$ and $f_{j}$ are independent for $\lambda$-almost all $j \in I .^{18}$

\footnotetext{
${ }^{17}$ All measures in this paper are countably additive set functions defined on $\sigma$-algebras.

${ }^{18}$ Two random variables $\phi$ and $\psi$ from $(\Omega, \mathcal{F}, P)$ to $X$ are said to be independent, if the $\sigma$-algebras $\sigma(\phi)$ and $\sigma(\psi)$ generated respectively by $\phi$ and $\psi$ are independent.
} 


\subsection{An exact law of large numbers for independent random full matchings}

We follow the notation in Section 3.1. Below is a formal definition of random full matching.

Definition 3 (Full matching)

1. Let $S=\{1,2, \ldots, K\}$ be a finite set of types, $\alpha: I \rightarrow S$ an $\mathcal{I}$-measurable type function of agents. Let $p$ denote the distribution on $S$. That is, for $1 \leq k \leq K$ and $I_{k}=\{i \in I$ : $\alpha(i)=k\}$, let $p_{k}=\lambda\left(I_{k}\right)$ for each $1 \leq k \leq K$.

2. A full matching $\phi$ is a bijection from $I$ to $I$ such that for each $i \in I, \phi(i) \neq i$ and $\phi(\phi(i))=i$.

3. A random full matching $\pi$ is a mapping from $I \times \Omega$ to $I$ such that (i) $\pi_{\omega}$ is a full matching for each $\omega \in \Omega$; (ii) let $g$ be the type process $\alpha(\pi)$; then $g$ is measurable from $(I \times \Omega, \mathcal{I} \otimes \mathcal{F}, \lambda \otimes P)$ to $S$; (iii) for $\lambda$-almost all $i \in I, g_{i}$ has distribution $p$.

4. A random full matching $\pi$ is said to be independent in types if the type process $g$ is essentially pairwise independent.

Condition (1) of this definition says that a fraction $p_{k}$ of the population is of type $k$. Condition (2) says that there is no self-matching, and that if $i$ is matched to $j=\phi(i)$, then $j$ is matched to $i$. Condition (3) (iii) means that for almost every agent $i$, the probability that $i$ is matched to a type- $k$ agent is $p_{k}$, the fraction of type- $k$ agents in the population. Condition (4) says that for almost all agents $i$ and $j \in I$, the event that agent $i$ matched to a type- $k$ agent is independent of the event that agent $j$ matched to a type- $l$ agent, for any $k$ and $l$ in $S$.

Because agents of type $k$ have a common probability $p_{l}$ of being matched to type- $l$ agents, Condition (4) allows the application of the exact law of large numbers in [45] and [48] (which is stated as Lemma 1 in Section 7.1 below) in order to claim that the relative fraction of agents matched to type- $l$ agents among the type- $k$ population is almost surely $p_{l}$ (or, intuitively, frequency coincides with probability). This means that the fraction of the total population consisting of type- $k$ agents that are matched to type- $l$ is almost surely $p_{k} \cdot p_{l}$. This result is formally stated in the following theorem, whose proof is given in Section 7.2.

Theorem 1 Let $\alpha: I \rightarrow S$ be an $\mathcal{I}$-measurable type function with type distribution $p=$ $\left(p_{1}, \ldots, p_{K}\right)$ on $S$. Let $\pi$ be a random full matching from $I \times \Omega$ to $I$. If $\pi$ is independent in types, then for any given types $(k, l) \in S \times S$,

$$
\lambda\left(\left\{i: \alpha(i)=k, \alpha\left(\pi_{\omega}(i)\right)=l\right\}\right)=p_{k} \cdot p_{l}
$$

holds for P-almost all $\omega \in \Omega$. 


\subsection{An exact law of large numbers for independent random partial matchings}

We shall now consider the case of random partial matchings, starting with the formal definition.

Definition 4 Let $\alpha: I \rightarrow S$ be an $\mathcal{I}$-measurable type function with type distribution $p=$ $\left(p_{1}, \ldots, p_{K}\right)$ on $S$. Let $\pi$ be a mapping from $I \times \Omega$ to $I \cup\{J\}$, where $J$ denotes "no match."

1. We say that $\pi$ is a random partial matching with no-match probabilities $q_{1}, \ldots, q_{K}$ in $[0,1]$ if (i) for each $\omega \in \Omega$, the restriction of $\pi_{\omega}$ to $I-\pi_{\omega}^{-1}(\{J\})$ is a full matching on $I-\pi_{\omega}^{-1}(\{J\}) i^{19}$ (ii) after extending the type function $\alpha$ to $I \cup\{J\}$ so that $\alpha(J)=J$, and letting $g=\alpha(\pi)$, we have $g$ measurable from $(I \times \Omega, \mathcal{I} \otimes \mathcal{F}, \lambda \otimes P)$ to $S \cup\{J\}$; (iii) for $\lambda$-almost all $i \in I_{k}, P\left(g_{i}=J\right)=q_{k}$ and $d^{20}$

$$
P\left(g_{i}=l\right)=\frac{\left(1-q_{k}\right) p_{l}\left(1-q_{l}\right)}{\sum_{r=1}^{K} p_{r}\left(1-q_{r}\right)} .
$$

2. A random partial matching $\pi$ is said to be independent in types if the process $g$ (taking values in $S \cup\{J\})$ is essentially pairwise independent. ${ }^{21}$

The following result, proved in Section 7.2, generalizes Theorem 1 to the case of random partial matchings.

Theorem 2 If $\pi$ is an independent-in-types random partial matching from $I \times \Omega$ to $I \cup\{J\}$ with no-match probabilities $q_{1}, \ldots, q_{K}$ then, for $P$-almost all $\omega \in \Omega$ :

1. The fraction of the total population consisting of unmatched agents of type $k$ is

$$
\lambda\left(\left\{i \in I: \alpha(i)=k, g_{\omega}(i)=J\right\}\right)=p_{k} q_{k} .
$$

2. For any types $(k, l) \in S^{2}$, the fraction of the total population consisting of type- $k$ agents that are matched to type-l agents is

$$
\lambda\left(\left\{i: \alpha(i)=k, g_{\omega}(i)=l\right\}\right)=\frac{p_{k}\left(1-q_{k}\right) p_{l}\left(1-q_{l}\right)}{\sum_{r=1}^{K} p_{r}\left(1-q_{r}\right)} .
$$

\footnotetext{
${ }^{19}$ This means that an agent $i$ with $\pi_{\omega}(i)=J$ is not matched, while any agent in $I-\pi_{\omega}^{-1}(\{J\})$ is matched. This produces a partial matching on $I$.

${ }^{20}$ Note that if an agent of type $k$ is matched, its probability of being matched to a type- $l$ agent should be proportional to the type distribution of matched agents. The fraction of the population of matched agents among the total population is $\sum_{r=1}^{K} p_{r}\left(1-q_{r}\right)$. Thus, the relative fraction of type $l$ matched agents to that of all the matched agents is $\left(p_{l}\left(1-q_{l}\right)\right) / \sum_{r=1}^{K} p_{r}\left(1-q_{r}\right)$. This implies that the probability that a type- $k$ agent is matched to a type- $l$ agent is $\left(1-q_{k}\right)\left(p_{l}\left(1-q_{l}\right)\right) / \sum_{r=1}^{K} p_{r}\left(1-q_{r}\right)$. When $\sum_{r=1}^{K} p_{r}\left(1-q_{r}\right)=0$, we have $p_{k}\left(1-q_{k}\right)=0$ for all $1 \leq k \leq K$, in which case almost no agents are matched, and we can interpret the ratio $\left(\left(1-q_{k}\right) p_{l}\left(1-q_{l}\right)\right) / \sum_{r=1}^{K} p_{r}\left(1-q_{r}\right)$ as zero.

${ }^{21}$ This means that for almost all agents $i, j \in I$, whether agent $i$ is unmatched or matched to a type- $k$ agent is independent of a similar event for agent $j$.
} 


\section{A dynamical system with random mutation, partial matching, and type changing that is Markov conditionally independent in types}

In this section, we consider a dynamical system with random mutation, partial matching and type changing that is Markov conditionally independent in types. We first define such a dynamical system in Section 4.1. Then, we formulate in Section 4.2 the key condition of Markov conditional independence in types, and finally present in Theorem 3 of Section 4.3 an exact law of large numbers and stationarity for the dynamical system.

\subsection{Definition of a dynamical system with random mutation, partial matching and type changing}

Let $S=\{1,2, \ldots, K\}$ be a finite set of types. A discrete-time dynamical system $\mathbb{D}$ with random mutation, partial matching and type changing in each period can be defined intuitively as follows. The initial distribution of types is $p^{0}$. That is, $p^{0}(k)$ (denoted by $p_{k}^{0}$ ) is the initial fraction of agents of type $k$. In each time period, each agent of type $k$ first goes through a stage of random mutation, becoming an agent of type $l$ with probability $b_{k l}$. In models such as [15], for example, this mutation generates new motives for trade. Then, each agent of type $k$ is either not matched, with probability $q_{k}$, or is matched to a type- $l$ agent with a probability proportional to the fraction of type- $l$ agents in the population immediately after the random mutation step. When an agent is not matched, she keeps her type. Otherwise, when a pair of agents with respective types $k$ and $l$ are matched, each of the two agents changes types; the type- $k$ agent becomes type $r$ with probability $\nu_{k l}(r)$, where $\nu_{k l}$ is a probability distribution on $S$, and similarly for the type- $l$ agent. Under appropriate independence conditions, one would like to have an almost-surely deterministic cross-sectional type distribution at each time period.

We shall now define formally a dynamical system $\mathbb{D}$ with random mutation, partial matching and type changing. As in Section 3 , let $(I, \mathcal{I}, \lambda)$ be an atomless probability space representing the space of agents, $(\Omega, \mathcal{F}, P)$ a sample probability space, and $(I \times \Omega, \mathcal{I} \otimes \mathcal{F}, \lambda \otimes P)$ a Fubini extension of the usual product probability space.

Let $\alpha^{0}: I \rightarrow S=\{1, \ldots, K\}$ be an initial $\mathcal{I}$-measurable type function with distribution $p^{0}$ on $S$. For each time period $n \geq 1$, we first have a random mutation that is modeled by a process $h^{n}$ from $(I \times \Omega, \mathcal{I} \otimes \mathcal{F}, \lambda \otimes P)$ to $S$, then a random partial matching that is described by a function $\pi^{n}$ from $(I \times \Omega, \mathcal{I} \otimes \mathcal{F}, \lambda \otimes P)$ to $I \cup\{J\}$ (where $J$ represents no matching), followed by type changing for the matched agents that is modeled by a process $\alpha^{n}$ from $(I \times \Omega, \mathcal{I} \otimes \mathcal{F}, \lambda \otimes P)$ to $S$.

For the random mutation step at time $n$, given a $K \times K$ probability transition matrix ${ }^{22}$

\footnotetext{
${ }^{22}$ Here, $b_{k l}$ is in $[0,1]$, with $\sum_{l=1}^{K} b_{k l}=1$ for each $k$.
} 
$b$, we require that, for each agent $i \in I$,

$$
P\left(h_{i}^{n}=l \mid \alpha_{i}^{n-1}=k\right)=b_{k l}
$$

the specified probability with which an agent $i$ of type $k$ at the end of time period $n-1$ mutates to type $l$.

For the random partial matching step at time $n$, we let $\bar{p}^{n-1 / 2}$ be the expected crosssectional type distribution immediately after random mutation. That is,

$$
\bar{p}_{k}^{n-1 / 2}=\bar{p}^{n-1 / 2}(k)=\int_{\Omega} \lambda\left(\left\{i \in I: h_{\omega}^{n}(i)=k\right\}\right) d P(\omega) .
$$

The random partial matching function $\pi^{n}$ at time $n$ is defined by:

1. For any $\omega \in \Omega, \pi_{\omega}^{n}(\cdot)$ is a full matching on $I-\left(\pi_{\omega}^{n}\right)^{-1}(\{J\})$, as defined in Section 3.3.

2. Extending $h^{n}$ so that $h^{n}(J, \omega)=J$ for any $\omega \in \Omega$, we define $g^{n}: I \times \Omega \rightarrow S \cup\{J\}$ by

$$
g^{n}(i, \omega)=h^{n}\left(\pi^{n}(i, \omega), \omega\right)
$$

and assume that $g^{n}$ is $\mathcal{I} \otimes \mathcal{F}$-measurable.

3. Let $q \in[0,1]^{S}$. For each agent $i \in I$,

$$
\begin{aligned}
P\left(g_{i}^{n}=J \mid h_{i}^{n}=k\right) & =q_{k}, \\
P\left(g_{i}^{n}=l \mid h_{i}^{n}=k\right) & =\frac{\left(1-q_{k}\right)\left(1-q_{l}\right) \bar{p}_{l}^{n-1 / 2}}{\sum_{r=1}^{K}\left(1-q_{r}\right) \bar{p}_{r}^{n-1 / 2}} .
\end{aligned}
$$

Equation (10) means that, for any agent whose type before the matching is $k$, the probability of being unmatched is $q_{k}$, and the probability of being matched to a type- $l$ agent is proportional to the expected cross-sectional type distribution for matched agents. When $g^{n}$ is essentially pairwise independent (as under the Markov conditional independence condition used in Section 4.3 below), the exact law of large numbers in [45] and [48] (see Lemma 1 below) implies that the realized cross-sectional type distribution $\lambda\left(h_{\omega}^{n}\right)^{-1}$ after random mutation at time $n$ is indeed the expected distribution $\bar{p}^{n-1 / 2}, P$-almost surely. ${ }^{23}$

Finally, for the step of random type changing for matched agents at time $n$, a given $\nu: S \times S \rightarrow \Delta$ specifies the probability distribution $\nu_{k l}=\nu(k, l)$ of the new type of a type- $k$ agent who has met a type- $l$ agent. When agent $i$ is not matched at time $n$, she keeps her type

\footnotetext{
${ }^{23}$ As noted in Footnote 20, if the denominator in equation (10) is zero, then almost no agents will be matched and we can simply interpret the ratio as zero.
} 
$h_{i}^{n}$ with probability one. We thus require that the type function $\alpha^{n}$ after matching satisfies, for each agent $i \in I$,

$$
\begin{aligned}
P\left(\alpha_{i}^{n}=r \mid h_{i}^{n}=k, g_{i}^{n}=J\right) & =\delta_{k}^{r}, \\
P\left(\alpha_{i}^{n}=r \mid h_{i}^{n}=k, g_{i}^{n}=l\right) & =\nu_{k l}(r),
\end{aligned}
$$

where $\delta_{k}^{r}$ is one if $r=k$, and zero otherwise.

Thus, we have inductively defined a dynamical system $\mathbb{D}$ with random mutation, partial matching, and match-induced type changing with parameters $\left(p^{0}, b, q, \nu\right)$.

\subsection{Markov conditional independence in types}

In this section, we consider a suitable independence condition on the dynamical system $\mathbb{D}$. For $n \geq 1$, to formalize the intuitive idea that given their type function $\alpha^{n-1}$, the agents randomly mutate to other types independently at time $n$, and that their types in earlier periods have no effect on this mutation, we say that the random mutation is Markov conditionally independent in types if, for $\lambda$-almost all $i \in I$ and $\lambda$-almost all $j \in I$,

$$
P\left(h_{i}^{n}=k, h_{j}^{n}=l \mid \alpha_{i}^{0}, \ldots, \alpha_{i}^{n-1} ; \alpha_{j}^{0}, \ldots, \alpha_{j}^{n-1}\right)=P\left(h_{i}^{n}=k \mid \alpha_{i}^{n-1}\right) P\left(h_{j}^{n}=l \mid \alpha_{j}^{n-1}\right)
$$

holds for all types $k, l \in S .{ }^{24}$

Intuitively, the random partial matching at time $n$ should depend only on agents' types immediately after the random mutation. One may also want the random partial matching to be independent across agents, given events that occurred in the first $n-1$ time periods and the random mutation at time $n$. We say that the random partial matching $\pi^{n}$ is Markov conditionally independent in types if, for $\lambda$-almost all $i \in I$ and $\lambda$-almost all $j \in I$,

$$
P\left(g_{i}^{n}=c, g_{j}^{n}=d \mid \alpha_{i}^{0}, \ldots, \alpha_{i}^{n-1}, h_{i}^{n} ; \alpha_{j}^{0}, \ldots, \alpha_{j}^{n-1}, h_{j}^{n}\right)=P\left(g_{i}^{n}=c \mid h_{i}^{n}\right) P\left(g_{j}^{n}=d \mid h_{j}^{n}\right)
$$

holds for all types $c, d \in S \cup\{J\}$.

The agents' types at the end of time period $n$ should depend on the agents' types immediately after the random mutation stage at time $n$, as well as the results of random partial matching at time $n$, but not otherwise on events that occurred in previous periods. This motivates the following definition. The random type changing after partial matching at time $n$ is said to be Markov conditionally independent in types if for $\lambda$-almost all $i \in I$ and $\lambda$-almost all $j \in I$, and for each $n \geq 1$,

$$
\begin{aligned}
P\left(\alpha_{i}^{n}=k, \alpha_{j}^{n}\right. & \left.=l \mid \alpha_{i}^{0}, \ldots, \alpha_{i}^{n-1}, h_{i}^{n}, g_{i}^{n} ; \alpha_{j}^{0}, \ldots, \alpha_{j}^{n-1}, h_{j}^{n}, g_{j}^{n}\right) \\
& =P\left(\alpha_{i}^{n}=k \mid h_{i}^{n}, g_{i}^{n}\right) P\left(\alpha_{j}^{n}=l \mid h_{j}^{n}, g_{j}^{n}\right)
\end{aligned}
$$

\footnotetext{
${ }^{24}$ We could include the functions $h^{m}$ and $g^{m}$ for $1 \leq m \leq n-1$ as well. However, it is not necessary to do so since we only care about the dependence structure across time for the type functions at the end of each time period.
} 
holds for all types $k, l \in S$.

The dynamical system $\mathbb{D}$ is said to be Markov conditionally independent in types if, in each time period $n$, each random step (random mutation, partial matching, and type changing) is so.

\subsection{Exact law of large numbers and stationarity}

With the goal of a stationarity result for the cross-sectional type distribution, we now define a mapping $\Gamma$ from $\Delta$ to $\Delta$ such that, for each $p=\left(p_{1}, \ldots, p_{K}\right) \in \Delta$, the $r$-th component of $\Gamma$ is

$$
\Gamma_{r}\left(p_{1}, \ldots, p_{K}\right)=q_{r} \sum_{m=1}^{K} p_{m} b_{m r}+\sum_{k, l=1}^{K} \frac{\nu_{k l}(r)\left(1-q_{k}\right)\left(1-q_{l}\right) \sum_{m=1}^{K} p_{m} b_{m k} \sum_{j=1}^{K} p_{j} b_{j l}}{\sum_{t=1}^{K}\left(1-q_{t}\right) \sum_{j=1}^{K} p_{j} b_{j t}}
$$

We note that the second term of this expression for $\Gamma_{r}\left(p_{1}, \ldots, p_{K}\right)$ can be written as

$$
\sum_{k=1}^{K}\left(1-q_{k}\right) \sum_{m=1}^{K} p_{m} b_{m k} \frac{\sum_{l=1}^{K} \nu_{k l}(r)\left(1-q_{l}\right) \sum_{j=1}^{K} p_{j} b_{j l}}{\sum_{l=1}^{K}\left(1-q_{l}\right) \sum_{j=1}^{K} p_{j} b_{j l}}
$$

which is less than or equal to $\sum_{l=1}^{K}\left(1-q_{l}\right) \sum_{j=1}^{K} p_{j} b_{j l}$. This means that one can define $\Gamma_{r}\left(p_{1}, \ldots, p_{K}\right)$ to be $q_{r} \sum_{m=1}^{K} p_{m} b_{m r}$ when $\sum_{l=1}^{K}\left(1-q_{l}\right) \sum_{j=1}^{K} p_{j} b_{j l}=0$, in order to have continuity of $\Gamma$ on all of $\Delta$.

We let $p^{n}(\omega)_{k}=\lambda\left(\left\{i \in I: \alpha_{\omega}^{n}(i)=k\right\}\right)$ be the fraction of the population of type $k$ at the end of time period $n$ in state of nature $\omega$, and let $\bar{p}_{k}^{n}$ be it's expectation. That is,

$$
\bar{p}_{k}^{n}=\int_{\Omega} p^{n}(\omega) d P(\omega)=\int_{I} P\left(\alpha_{i}^{n}=k\right) d \lambda(i),
$$

where the last equality follows from the Fubini property.

The following theorem provides an exact law of large numbers and shows the stationarity for a dynamical system $\mathbb{D}$ with random mutation, partial matching, and type changing that is Markov conditionally independent in types. Its proof is given in Section 7.3.

Theorem 3 Let $\mathbb{D}$ be a dynamical system with random mutation, partial matching and type changing whose parameters are $\left(p^{0}, b, q, \nu\right)$. If $\mathbb{D}$ is Markov conditionally independent in types, then:

1. For each time $n \geq 1$, the expected cross-sectional type distribution is given by $\bar{p}^{n}=$ $\Gamma\left(\bar{p}^{n-1}\right)=\Gamma^{n}\left(p^{0}\right)$, and $\bar{p}_{k}^{n-1 / 2}=\sum_{l=1}^{K} b_{l k} \bar{p}_{l}^{n-1}$, where $\Gamma^{n}$ is the composition of $\Gamma$ with itself $n$ times, and where $\bar{p}^{n-1 / 2}$ is the expected cross-sectional type distribution after the random mutation (see equation (9)). 
2. For $\lambda$-almost all $i \in I,\left\{\alpha_{i}^{n}\right\}_{n=0}^{\infty}$ is a Markov chain with transition matrix $z^{n}$ time $n-1$ defined by

$$
z_{k l}^{n}=q_{l} b_{k l}+\sum_{r, j=1}^{K} \nu_{r j}(l) b_{k r} \frac{\left(1-q_{r}\right)\left(1-q_{j}\right) \bar{p}_{j}^{n-1 / 2}}{\sum_{r^{\prime}=1}^{K}\left(1-q_{r^{\prime}}\right) \bar{p}_{r^{\prime}}^{n-1 / 2}} .
$$

3. For $\lambda$-almost all $i \in I$ and $\lambda$-almost all $j \in I$, the Markov chains $\left\{\alpha_{i}^{n}\right\}_{n=0}^{\infty}$ and $\left\{\alpha_{j}^{n}\right\}_{n=0}^{\infty}$ are independent (which means that the random vectors $\left(\alpha_{i}^{0}, \ldots, \alpha_{i}^{n}\right)$ and $\left(\alpha_{j}^{0}, \ldots, \alpha_{j}^{n}\right)$ are independent for all $n \geq 0$ ).

4. For P-almost all $\omega \in \Omega$, the cross-sectional type process $\left\{\alpha_{\omega}^{n}\right\}_{n=0}^{\infty}$ is a Markov chain with transition matrix $z^{n}$ at time $n-1$.

5. For $P$-almost all $\omega \in \Omega$, at each time period $n \geq 1$, the realized cross-sectional type distribution after the random mutation $\lambda\left(h_{\omega}^{n}\right)^{-1}$ is its expectation $\bar{p}^{n-1 / 2}$, and the realized cross-sectional type distribution at the end of period $n, p^{n}(\omega)=\lambda\left(\alpha_{\omega}^{n}\right)^{-1}$, is equal to its expectation $\bar{p}^{n}$, and thus, P-almost surely, $p^{n}=\Gamma^{n}\left(p^{0}\right)$.

6. There is a stationary distribution $p^{*}$. That is, with initial cross-sectional type distribution $p^{0}=p^{*}$, for every $n \geq 1$, the realized cross-sectional type distribution $p^{n}$ at time $n$ is $p^{*} P$-almost surely, and $z^{n}=z^{1}$. In particular, all of the relevant Markov chains are time-homogeneous with a constant transition matrix having $p^{*}$ as a fixed point. ${ }^{25}$

\section{Existence of random matching models that are independent in types}

In this section, we state three existence results for independent random matching models in static and dynamic settings. These results are fully developed in a companion paper [17]. Propositions 1, 2 and 3 below are Theorems 2.4, 2.6 and 3.1 in [17] respectively.

The first result shows the existence of an independent random full matching model that satisfies a few strong conditions that are specified in Footnote 4 of McLennan and Sonnenschein [39], and is universal in the sense that it does not depend on particular type functions. ${ }^{26}$ Note

\footnotetext{
${ }^{25}$ Our initial type function $\alpha^{0}$ is assumed to be non-random. It is easy to generalize to the case in which $\alpha^{0}$ is a function from $I \times \Omega$ to $S$ such that for $\lambda$-almost all $i \in I, j \in I, \alpha_{i}^{0}$ and $\alpha_{j}^{0}$ are independent. Let $\bar{p}^{0}$ be the expected cross-sectional type distribution. Then, all the results in Theorem 3 remain valid. In the case that for $\lambda$-almost all $i \in I, \alpha_{i}^{0}$ has distribution $\bar{p}^{0}$, the evolution of the fractions of each type is essentially deterministic and coincides exactly with the evolution of the probability distribution of type for almost every given agent (in comparison with Footnote 15).

${ }^{26}$ When $(I, \mathcal{I}, \lambda)$ is taken to be the unit interval with the Borel algebra and Lebesgue measure, property (1) (iii) of Proposition 1 can be restated as "for $P$-almost all $\omega \in \Omega, \lambda\left(A_{1} \cap \pi_{\omega}^{-1}\left(A_{2}\right)\right)=\lambda\left(A_{1}\right) \lambda\left(A_{2}\right)$ holds for any $A_{1}, A_{2} \in \mathcal{I}$ " by using the fact that the countable collection of rational intervals in $[0,1]$ generates the Borel algebra. Footnote 4 of [39] shows the non-existence of a random full matching $\pi$ that satisfies (i)-(iii) of part (1). As noted on page 252 of [19], this also implies the non-existence of a random full matching model that is universal, similar to the non-existence result for a countable population considered earlier in Proposition 4 of $[5]$.
} 
that condition (1) (ii) below implies that for any $i, j \in I, P\left(\pi_{i}=j\right)=0$ since $\lambda(\{j\})=0$, which means that the probability that agent $i$ is matched with a given agent $j$ is zero.

Proposition 1 There exists a Fubini extension $(I \times \Omega, \mathcal{I} \otimes \mathcal{F}, \lambda \otimes P)$ of the usual product probability space with an atomless probability measure $\lambda$ and a random full matching $\pi$ from $(I \times \Omega, \mathcal{I} \otimes \mathcal{F}, \lambda \otimes P)$ to $I$ such that

1. (i) for each $\omega \in \Omega, \lambda\left(\pi_{\omega}^{-1}(A)\right)=\lambda(A)$ for any $A \in \mathcal{I}$, (ii) for each $i \in I, P\left(\pi_{i}^{-1}(A)\right)=$ $\lambda(A)$ for any $A \in \mathcal{I}$, (iii) for any $A_{1}, A_{2} \in \mathcal{I}, \lambda\left(A_{1} \cap \pi_{\omega}^{-1}\left(A_{2}\right)\right)=\lambda\left(A_{1}\right) \lambda\left(A_{2}\right)$ holds for $P$-almost all $\omega \in \Omega$;

2. $\pi$ is independent in types with respect to any given type function $\alpha$ from $I$ to any finite type space $S$.

Next, we consider a model for independent random partial matchings. Since the case of no-matching is type-dependent, it is not possible to produce a universal matching model for random partial matchings as in the case of full matchings.

Proposition 2 There is an atomless probability space $(I, \mathcal{I}, \lambda)$ of agents such that for any given $\mathcal{I}$-measurable type function $\beta$ from $I$ to $S$, and for any $q \in[0,1]^{S},(1)$ there exists a sample space $(\Omega, \mathcal{F}, P)$ and a Fubini extension $(I \times \Omega, \mathcal{I} \otimes \mathcal{F}, \lambda \otimes P)$ of the usual product probability space; (2) there exists an independent-in-types random partial matching $\pi$ from $(I \times \Omega, \mathcal{I} \otimes \mathcal{F}, \lambda \otimes P)$ to $I$ with $q=\left(q_{1}, \ldots, q_{K}\right)$ as the no-match probabilities.

Finally, we present a result on the existence of a dynamical system with random mutation, partial matching and type changing that is Markov conditionally independent in types.

Proposition 3 Fixing any parameters $p^{0}$ for initial cross-sectional type distribution, $b$ for $m u$ tation probabilities, $q \in[0,1]^{S}$ for no-match probabilities, and $\nu$ for match-induced type-change probabilities, there exists a Fubini extension of the usual product probability space on which is defined a dynamical system $\mathbb{D}$ with random mutation, partial matching and type changing that is Markov conditionally independent in types with these parameters $\left(p^{0}, b, q, \nu\right)$.

The static and dynamic matching models described in Propositions 1, 2, and 3 satisfy the respective conditions in Theorems 1,2 , and 3 . Thus, the respective conclusions in Theorems 1, 2 and 3 also hold for these matching models. 


\section{Discussion}

As noted in the introduction, this is the first theoretical treatment of the exact law of large numbers for independent random matching among a continuum population (modeled by an atomless, countably additive probability measure space). All three basic issues concerning independent random matching for a continuum population, namely, mathematical formulation of the analytic framework, proof of general results on the exact law of large numbers for independent random matching, and existence of independent random matching with the desired properties, are addressed for both static and dynamic systems. Our results on a dynamical system with random mutation, random partial matching, and random type changing provide an understanding of the time evolution of the cross-sectional type process, identifying it as a Markov chain with known transition matrices.

McLennan and Sonnenschein showed, in Footnote 4 of [39], the non-existence of a random full matching $\pi$ that satisfies a few strong conditions when $(I, \mathcal{I}, \lambda)$ is taken to be the unit interval with the Borel algebra and Lebesgue measure. As noted in Footnote 26, our Proposition 1 shows that the conditions of McLennan and Sonnenschein can be satisfied in our framework. There are also several negative results in the settings of [5, Proposition 4] and [19, Corollary 3.2] on the impossibility of finding a universal random matching that does not depend on particular type functions. Proposition 1, however, shows that a universal random full matching does exist in our framework.

Based on the classical asymptotic law of large numbers, Boylan constructed an example of random full matching for a countable population in [5, Proposition 2] with the properties that an individual's probability of matching a type- $k$ agent is the fraction $p_{k}$ of type- $k$ agents in the total population, and that the asymptotic fraction of type- $k$ agents matching type- $l$ agents in a realized matching approximates $p_{k} p_{r}$ almost surely. ${ }^{27} \mathrm{~A}$ repeated matching scheme is then considered in [5] for the dynamic setting.

An ad-hoc example of random full matching is also constructed in [19, Theorem 4.2] for a given type function on the population space $[0,1]$ by rearranging intervals in $[0,1]$ through measure-preserving mappings. For repeated matching schemes with an infinite number of time periods, it is recognized in [19, page 262] that one may run into problems when the matching in the next period follows from the type function in a previous period. ${ }^{28}$ It is then proposed to arbitrarily rearrange agents with the same types into half-open intervals. Aside from the question of a natural interpretation of this rearrangement of agents' names using intervals,

\footnotetext{
${ }^{27}$ It is not clear whether this example satisfies the kind of condition, independence in types, considered by us.

${ }^{28}$ In the dynamic random matching model defined in the proof of our Proposition 3 in [17], every step of randomization uses the realized type function generated in the step of randomization immediately before.
} 
the random full matching considered in [19] does not satisfy the intuitive idea that agents are matched independently in types. That is, this example is not a model for independent random matching. It is made clear in [19, page 266] that "This paper should not be viewed as a justification for the informal use of a law of large numbers in random matching with a continuum of agents."

In comparison with the particular examples of a random full matching with some matching properties in [5] and [19], we prove the exact law of large numbers for general independent random matchings, ${ }^{29}$ which can be applied to different matching schemes. Our existence result in Proposition 1 also has stronger properties than those in [5, Proposition 2] and [19, Theorem 4.2]. Also, random mutation, random partial matching and random type changing induced by matching are not considered in [5] and [19].

Gilboa and Matsui [23] constructed a particular example for a matching model of two countable populations with a countable number of encounters in the time interval $[0,1)$, where the space $\mathbb{N}$ of agents is endowed with a purely finitely additive measure $\mu$ extending the usual density. They showed that their matching model satisfies a few desired matching properties in their setting, including the fact that an agent is matched exactly once with probability one. Their matching model is quite different from ours. As they also point out, a disadvantage of their approach is that the underlying state of the world is "drawn" according to a purely finitelyadditive measure. In addition to the fact that a purely finitely-additive measure-theoretic framework does not allow the use of analytic tools involving limits, it has no basic properties, such as Radon-Nikodym derivatives, and the property that everywhere positive, integrable functions having positive integrals. ${ }^{30}$ In short, one cannot use probabilistic arguments in such a framework. Moreover, as in Section 6.2 of [48], absurd results can be obtained in a purely finitely additive settings. For example, for an iid sequence of random variables defined on a purely finitely additive measure space, one can say that the arithmetic averages of almost all sample sequences diverge (or converge to any given value).

As emphasized earlier, we have shown in Theorem 3 that the cross-sectional type process defined on our agent space $(I, \mathcal{I}, \lambda)$ (which is a countably additive atomless probability space) is a Markov chain with known transition matrices almost surely. It would not make sense to have such a result when the space of agents is $\mathbb{N}$ endowed with a purely finitely additive measure $\mu$. Note that a Markov chain $\left\{\psi_{n}\right\}_{n=0}^{\infty}$ on $S$ in general induces an atomless countably additive

\footnotetext{
${ }^{29}$ It is important to distinguish an ad hoc example without independence from a general result in the setting of law of large numbers. For example, one can take a sequence of bounded random variables $\left\{\phi_{n}\right\}_{n=1}^{\infty}$ with mean zero. When all the odd terms in the sequence equal $\phi_{1}$ and even terms equal $-\phi_{1}$, then $\left(\sum_{k=1}^{n} \phi_{k}\right) / n$ converges to zero almost surely. Such kind of result will not be useful at all in situations that require the use of the law of large numbers.

${ }^{30}$ In some sense, the weights for a purely finitely additive measure may be concentrated at an imaginary infinity; see [3].
} 
measure on $S^{\infty}$, which cannot be induced by any mapping from $\mathbb{N}$ with the measure $\mu$ to $S^{\infty}$.

\section{Appendix}

\subsection{Exact law of large numbers for a continuum of independent random variables}

The following general version of the exact law of large numbers is shown by Sun in [45] and [48], and is stated as a lemma here for the convenience of the reader. ${ }^{31}$

Lemma 1 Let $f$ be a process from $(I \times \Omega, \mathcal{I} \otimes \mathcal{F}, \lambda \otimes P)$ to a complete separable metric space $X$. Assume that the random variables $f_{i}$ are essentially pairwise independent.

1. For P-almost all $\omega \in \Omega$, the sample distribution $\lambda f_{\omega}^{-1}$ of the sample function $f_{\omega}$ is the same as the distribution $(\lambda \otimes P) f^{-1}$ of the process. ${ }^{32}$

2. For any $A \in \mathcal{I}$ with $\lambda(A)>0$, let $f^{A}$ be the restriction of $f$ to $A \times \Omega, \lambda^{A}$ and $\lambda^{A} \otimes P$ the probability measures rescaled from the restrictions $\lambda$ and $\lambda \otimes P$ to $\{D \in \mathcal{I}: D \subseteq A\}$ and $\{C \in \mathcal{I} \otimes \mathcal{F}: C \subseteq A \times \Omega\}$ respectively. Then for $P$-almost all $\omega \in \Omega$, the sample distribution $\lambda^{A}\left(f^{A}\right)_{\omega}^{-1}$ of the sample function $\left(f^{A}\right)_{\omega}$ is the same as the distribution of $\left(\lambda^{A} \otimes P\right)\left(f^{A}\right)^{-1}$ of the process $f^{A}$.

3. If there is a distribution $\mu$ on $X$ such that for $\lambda$-almost all $i \in I$, the random variable $f_{i}$ has distribution $\mu$, then the sample function $f_{\omega}\left(\right.$ or $\left.\left(f^{A}\right)_{\omega}\right)$ also has distribution $\mu$ for $P$-almost all $\omega \in \Omega$.

By viewing a discrete-time stochastic process taking values in $X$ as a random variable taking values in $X^{\infty}$, Lemma 1 implies the following exact law of large numbers for a continuum of discrete-time stochastic processes, which is formally stated in Theorem 5.8 in [45] and Theorem 3.13 in [48] respectively. ${ }^{33}$

\footnotetext{
${ }^{31}$ This result was originally stated on Loeb measure spaces in [45] (Theorem 5.2). However, it is noted in [48] that the result can be proved for an extension of the usual product with the Fubini property (Theorem 3.5); see also Chapter 7 in [37] (and in particular, Section 7.5), written by Sun. Theorem 3.5 of [48] actually shows that the statement in (2) here is equivalent to the condition of essential pairwise independence, which simply presents Theorem 7.6 in the earlier paper [45] in the Loeb space framework to the setting of a Fubini extension of the usual product probability space.

${ }^{32}$ Here, $(\lambda \otimes P) f^{-1}$ is the distribution $\nu$ on $X$ such that $\nu(B)=(\lambda \otimes P)\left(f^{-1}(B)\right)$ for any Borel set $B$ in $X$; $\lambda f_{\omega}^{-1}$ is defined similarly.

${ }^{33}$ For any given two Loeb spaces $(I, \mathcal{I}, \lambda)$ and $(\Omega, \mathcal{F}, P)$ as in [36] and [37], Anderson noted in [1] that the Loeb product space $(I \times \Omega, \mathcal{I} \otimes \mathcal{F}, \lambda \otimes P)$ is an extension of the usual product $(I \times \Omega, \mathcal{I} \otimes \mathcal{F}, \lambda \otimes P)$. Keisler proved in [31] (see also [37]) that the Fubini property still holds on $(I \times \Omega, \mathcal{I} \otimes \mathcal{F}, \lambda \otimes P)$. Thus, the Loeb product space is a Fubini extension of the usual product probability space. In addition, it is shown in Theorem 6.2 of [45] that when both $\lambda$ and $P$ are atomless, $(I \times \Omega, \mathcal{I} \otimes \mathcal{F}, \lambda \otimes P)$ is rich enough to be endowed with a process $h$ whose random variables are essentially pairwise independent and can take any variety of distributions (and in particular the uniform distribution on $[0,1])$. A countable infinite product of Loeb transition probabilities (which is not a Loeb product space) is used in [17] to construct a continuum of independent Markov chains that
} 
Corollary 1 Let $f$ be a mapping from $I \times \Omega \times \mathbb{N}$ to a complete separable metric space $X$ such that for each $n \geq 0, f^{n}=f(\cdot, \cdot, n)$ is an $\mathcal{I} \otimes \mathcal{F}$-measurable process. Then, for $\lambda$-almost all $i \in I$, $\left\{f_{i}^{n}\right\}_{n=0}^{\infty}$ is a discrete-time stochastic process. Assume that the stochastic processes $\left\{f_{i}^{n}\right\}_{n=0}^{\infty}, i \in$ $I$ are essentially pairwise independent, i.e., for $\lambda$-almost all $i \in I, \lambda$-almost all $j \in I$, the random vectors $\left(f_{i}^{0}, \ldots, f_{i}^{n}\right)$ and $\left(f_{j}^{0}, \ldots, f_{j}^{n}\right)$ are independent for all $n \geq 0$. Then, for $P$-almost all $\omega \in \Omega$, the empirical process $f_{\omega}=\left\{f_{\omega}^{n}\right\}_{n=0}^{\infty}$ has the same finite-dimensional distributions as that of $f=\left\{f^{n}\right\}_{n=0}^{\infty}$, i.e. $\left(f_{\omega}^{0}, \ldots, f_{\omega}^{n}\right)$ and $\left(f^{0}, \ldots, f^{n}\right)$ have the same distribution for any $n \geq 0$.

\subsection{Proofs of Theorems 1 and 2}

Proof of Theorem 1: If $p_{k}=0$, equation (5) is automatically satisfied. Consider $p_{k}>0$. Let $I_{k}=\{i \in I: \alpha(i)=k\}$ and $g=\alpha(\pi)$. Since the random variables $g_{i}$ are essentially pairwise independent, Lemma 1 (3) implies that the sample function $\left(g^{I_{k}}\right)_{\omega}$ on $I_{k}$ has distribution $p$ on $S$ for $P$-almost all $\omega \in \Omega$. This means that $\lambda\left(\left\{i \in I_{k}: g_{\omega}(i)=l\right\}\right) / p_{k}=p_{l}$ for $P$-almost all $\omega \in \Omega$. Hence equation (5) follows.

Proof of Theorem 2: The proof is similar to that of Theorem 1; we adopt the same notation and consider only $p_{k}>0$. Lemma 1 says that for $P$-almost all $\omega \in \Omega$, the sample function $g_{\omega}^{I_{k}}$ on $I_{k}$ has the same distribution as $g^{I_{k}}$ on $I_{k} \times \Omega$. Hence for $P$-almost all $\omega \in \Omega$,

$$
\lambda^{I_{k}}\left(\left(g_{\omega}^{I_{k}}\right)^{-1}(\{J\})\right)=\left(\lambda^{I_{k}} \otimes P\right)\left(\left(g^{I_{k}}\right)^{-1}(\{J\})\right),
$$

which means that

$$
\lambda\left(\left\{i \in I: \alpha(i)=k, g_{\omega}(i)=J\right\}\right)=\int_{I_{k}} \int_{\Omega} 1_{\left(g_{i}=J\right)} d P d \lambda=\int_{I_{k}} q_{k} d \lambda=p_{k} q_{k} ;{ }^{34}
$$

and also for any $1 \leq l \leq K$,

$$
\begin{aligned}
\lambda\left(I_{k} \cap g_{\omega}^{-1}(\{l\})\right) & =(\lambda \otimes P)\left(\left(I_{k} \times \Omega\right) \cap g^{-1}(\{l\})\right)=\int_{I_{k}} \int_{\Omega} 1_{\left(g_{i}=l\right)} d P d \lambda \\
& =\int_{I_{k}} \frac{\left(1-q_{k}\right) p_{l}\left(1-q_{l}\right)}{\sum_{r=1}^{K} p_{r}\left(1-q_{r}\right)} d \lambda=\frac{p_{k}\left(1-q_{k}\right) p_{l}\left(1-q_{l}\right)}{\sum_{r=1}^{K} p_{r}\left(1-q_{r}\right)} .
\end{aligned}
$$

Thus, equations (6) and (7) follow.

\subsection{Proof of Theorem 3}

Before proving Theorem 3, we need to prove a few lemmas. The first lemma shows how to compute the expected cross-sectional type distributions $\bar{p}^{n}$ and $\bar{p}^{n-1 / 2}$.

is derived from random mutation, random partial matching and random type changing. This means that though a Loeb product space is already very rich, we still need to work with the more general Fubini extension in some cases.

${ }^{34}$ For a set $C$ in a space, $1_{C}$ denotes its indicator function. 
Lemma 2 (1) For each $n \geq 1, \bar{p}^{n}=\Gamma\left(\bar{p}^{n-1}\right)$, and hence $\bar{p}^{n}=\Gamma^{n}\left(p^{0}\right)$, where $\Gamma^{n}$ is the composition of $\Gamma$ with itself $n$ times.

(2) For each $n \geq 1$, the expected cross-sectional type distribution $\bar{p}^{n-1 / 2}$ immediately after random mutation at time $n$, as defined in equation (9), satisfies $\bar{p}_{k}^{n-1 / 2}=\sum_{l=1}^{K} b_{l k} \bar{p}_{l}^{n-1}=$ $\sum_{l=1}^{K} b_{l k} \Gamma^{n-1}\left(p^{0}\right)$.

Proof. Equations (8) and (9) and the Fubini property imply that

$$
\begin{aligned}
\bar{p}_{k}^{n-1 / 2} & =\int_{I} P\left(h_{i}^{n}=k\right) d \lambda(i)=\int_{I} \sum_{l=1}^{K} P\left(h_{i}^{n}=k, \alpha_{i}^{n-1}=l\right) d \lambda(i) \\
& =\int_{I} \sum_{l=1}^{K} P\left(h_{i}^{n}=k \mid \alpha_{i}^{n-1}=l\right) P\left(\alpha_{i}^{n-1}=l\right) d \lambda(i) \\
& =\sum_{l=1}^{K} \int_{I} b_{l k} P\left(\alpha_{i}^{n-1}=l\right) d \lambda(i)=\sum_{l=1}^{K} b_{l k} \bar{p}_{l}^{n-1} .
\end{aligned}
$$

Then, we can express $\bar{p}^{n}$ in terms of $\bar{p}^{n-1 / 2}$ by equations (10) and (11).

$$
\begin{aligned}
\bar{p}_{r}^{n}= & \int_{I} P\left(\alpha_{i}^{n}=r\right) d \lambda(i) \\
= & \int_{I} \sum_{k=1}^{K}\left[P\left(\alpha_{i}^{n}=r, h_{i}^{n}=k, g_{i}^{n}=J\right)+\sum_{l=1}^{K} P\left(\alpha_{i}^{n}=r, h_{i}^{n}=k, g_{i}^{n}=l\right)\right] d \lambda(i) \\
= & \int_{I} \sum_{k=1}^{K}\left[P\left(\alpha_{i}^{n}=r \mid h_{i}^{n}=k, g_{i}^{n}=J\right) P\left(g_{i}^{n}=J \mid h_{i}^{n}=k\right) P\left(h_{i}^{n}=k\right)\right. \\
& \left.+\sum_{l=1}^{K} P\left(\alpha_{i}^{n}=r \mid h_{i}^{n}=k, g_{i}^{n}=l\right) P\left(g_{i}^{n}=l \mid h_{i}^{n}=k\right) P\left(h_{i}^{n}=k\right)\right] d \lambda(i) \\
= & \bar{p}_{r}^{n-1 / 2} q_{r}+\sum_{k, l=1}^{K} \frac{\nu_{k l}(r) \bar{p}_{k}^{n-1 / 2}\left(1-q_{k}\right) \bar{p}_{l}^{n-1 / 2}\left(1-q_{l}\right)}{\sum_{t=1}^{K} \bar{p}_{t}^{n-1 / 2}\left(1-q_{t}\right)} .
\end{aligned}
$$

By combining equations (18) and (19), it is easy to see that $\bar{p}^{n}=\Gamma\left(\bar{p}^{n-1}\right)$, and hence that $\bar{p}^{n}=\Gamma^{n}\left(p^{0}\right)$, where $\Gamma^{n}$ is the composition of $\Gamma$ with itself $n$ times. Hence, part (1) of the lemma is shown. Part (2) of the lemma follows from part (1) and equation (18).

The following lemma shows the Markov property of the agents' type processes.

Lemma 3 Suppose the dynamical system $\mathbb{D}$ is Markov conditionally independent in types. Then, for $\lambda$-almost all $i \in I$, the type process for agent $i,\left\{\alpha_{i}^{n}\right\}_{n=0}^{\infty}$, is a Markov chain with transition matrix $z^{n}$ at time $n-1$, where $z_{k l}^{n}$ is defined in equation (17).

Proof. Fix $n \geq 1$. Equation (12) implies that for $\lambda$-almost all $i \in I, \lambda$-almost all $j \in I$,

$$
\begin{aligned}
P\left(h_{i}^{n}=k_{n}, h_{j}^{n}\right. & \left.\in S \mid \alpha_{i}^{0}=k_{0}, \ldots, \alpha_{i}^{n-1}=k_{n-1} ; \alpha_{j}^{0} \in S, \ldots, \alpha_{j}^{n-1} \in S\right) \\
& =P\left(h_{i}^{n}=k_{n} \mid \alpha_{i}^{n-1}=k_{n-1}\right) P\left(h_{j}^{n} \in S \mid \alpha_{j}^{n-1}\right),
\end{aligned}
$$


holds for any $\left(k_{0}, \ldots, k_{n}\right) \in S^{n+1}$. Thus, for $\lambda$-almost all $i \in I$,

$$
P\left(h_{i}^{n}=k \mid \alpha_{i}^{0}, \ldots, \alpha_{i}^{n-1}\right)=P\left(h_{i}^{n}=k \mid \alpha_{i}^{n-1}\right)
$$

holds for any $k \in S$. By grouping countably many null sets together, we know that for $\lambda$-almost all $i \in I$, equation (21) holds for all $k \in S$ and $n \geq 1$.

Similarly, equations (13) and (14) imply that for $\lambda$-almost all $i \in I$,

$$
\begin{aligned}
P\left(g_{i}^{n}=c \mid \alpha_{i}^{0}, \ldots, \alpha_{i}^{n-1}, h_{i}^{n}\right) & =P\left(g_{i}^{n}=c \mid h_{i}^{n}\right) \\
P\left(\alpha_{i}^{n}=k \mid \alpha_{i}^{0}, \ldots, \alpha_{i}^{n-1}, h_{i}^{n}, g_{i}^{n}\right) & =P\left(\alpha_{i}^{n}=k \mid h_{i}^{n}, g_{i}^{n}\right)
\end{aligned}
$$

hold for all $k \in S, c \in S \cup\{J\}$ and $n \geq 1$. A simple computation shows that for $\lambda$-almost all $i \in I, P\left(\alpha_{i}^{n}=k \mid \alpha_{i}^{0}, \ldots, \alpha_{i}^{n-1}\right)=P\left(\alpha_{i}^{n}=k \mid \alpha_{i}^{n-1}\right)$ for all $k \in S$ and $n \geq 1$. Hence, for $\lambda$-almost all $i \in I$, agent $i$ 's type process $\left\{\alpha_{i}^{n}\right\}_{n=0}^{\infty}$ is a Markov chain; it is also easy to see that the transition matrix $z^{n}$ from time $n-1$ to time $n$ is

$$
\begin{aligned}
z_{k l}^{n} & =P\left(\alpha_{i}^{n}=l \mid \alpha_{i}^{n-1}=k\right) \\
& =\sum_{r=1}^{K} \sum_{c \in S \cup\{J\}} P\left(\alpha_{i}^{n}=l \mid h_{i}^{n}=r, g_{i}^{n}=c\right) P\left(g_{i}^{n}=c \mid h_{i}^{n}=r\right) P\left(h_{i}^{n}=r \mid \alpha_{i}^{n-1}=k\right) .
\end{aligned}
$$

Then, equations (8), (10) and (11) imply that the formula for $z_{k l}^{n}$ in equation (17) holds.

Now, for each $n \geq 1$, we view each $\alpha^{n}$ as a random variable on $I \times \Omega$. Then $\left\{\alpha^{n}\right\}_{n=0}^{\infty}$ is a discrete-time stochastic process.

Lemma 4 Assume that the dynamical system $\mathbb{D}$ is Markov conditionally independent in types. Then, $\left\{\alpha^{n}\right\}_{n=0}^{\infty}$ is also a Markov chain with transition matrix $z^{n}$ at time $n-1$ given by equation $(17)$.

Proof. We can compute the transition matrix of $\left\{\alpha^{n}\right\}_{n=0}^{\infty}$ at time $n-1$ as follows. For any $k, l \in S$, we have

$$
\begin{aligned}
(\lambda \otimes P)\left(\alpha^{n}=l, \alpha^{n-1}=k\right) & =\int_{I} P\left(\alpha_{i}^{n}=l \mid \alpha_{i}^{n-1}=k\right) P\left(\alpha_{i}^{n-1}=k\right) d \lambda(i) \\
& =\int_{I} z_{k l}^{n} P\left(\alpha_{i}^{n-1}=k\right) d \lambda(i) \\
& =z_{k l}^{n} \cdot(\lambda \otimes P)\left(\alpha^{n-1}=k\right),
\end{aligned}
$$

which implies that $(\lambda \otimes P)\left(\alpha^{n}=l \mid \alpha^{n-1}=k\right)=z_{k l}^{n}$.

Next, for any $n \geq 1$, and for any $\left(a^{0}, \ldots, a^{n-2}\right) \in S^{n-1}$, we have

$$
(\lambda \otimes P) \quad\left(\quad\left(\alpha^{0}, \ldots, \alpha^{n-2}\right)=\left(a^{0}, \ldots, a^{n-2}\right), \alpha^{n-1}=k, \alpha^{n}=l\right)
$$




$$
\begin{aligned}
& =\int_{I} P\left(\left(\alpha_{i}^{0}, \ldots, \alpha_{i}^{n-2}\right)=\left(a^{0}, \ldots, a^{n-2}\right), \alpha_{i}^{n-1}=k, \alpha_{i}^{n}=l\right) d \lambda(i) \\
& =\int_{I} P\left(\alpha_{i}^{n}=l \mid \alpha_{i}^{n-1}=k\right) P\left(\left(\alpha_{i}^{0}, \ldots, \alpha_{i}^{n-2}\right)=\left(a^{0}, \ldots, a^{n-2}\right), \alpha_{i}^{n-1}=k\right) d \lambda(i) \\
& =z_{k l}^{n} \cdot(\lambda \otimes P)\left(\left(\alpha^{0}, \ldots, \alpha^{n-2}\right)=\left(a^{0}, \ldots, a^{n-2}\right), \alpha^{n-1}=k\right),
\end{aligned}
$$

which implies that $(\lambda \otimes P)\left(\alpha^{n}=l \mid\left(\alpha^{0}, \ldots, \alpha^{n-2}\right)=\left(a^{0}, \ldots, a^{n-2}\right), \alpha^{n-1}=k\right)=z_{k l}^{n}$. Hence the discrete-time process $\left\{\alpha^{n}\right\}_{n=0}^{\infty}$ is indeed a Markov chain with transition matrix $z^{n}$ at time $n-1$.

To prove that the agents' type processes are essentially pairwise independent in Lemma 6 below, we need the following elementary lemma.

Lemma 5 Let $\phi_{m}$ be a random variable from $(\Omega, \mathcal{F}, P)$ to a finite space $A_{m}$, for $m=1,2,3,4$. If the random variables $\phi_{3}$ and $\phi_{4}$ are independent, and if, for all $a_{1} \in A_{1}$ and $a_{2} \in A_{2}$,

$$
P\left(\phi_{1}=a_{1}, \phi_{2}=a_{2} \mid \phi_{3}, \phi_{4}\right)=P\left(\phi_{1}=a_{1} \mid \phi_{3}\right) P\left(\phi_{2}=a_{2} \mid \phi_{4}\right),
$$

then the two pairs of random variables $\left(\phi_{1}, \phi_{3}\right)$ and $\left(\phi_{2}, \phi_{4}\right)$ are independent.

Proof. For any $a_{m} \in A_{m}, m=1,2,3,4$, we have

$$
\begin{aligned}
P\left(\phi_{1}=a_{1}, \phi_{2}=a_{2}, \phi_{3}\right. & \left.=a_{3}, \phi_{4}=a_{4}\right) \\
& =P\left(\phi_{1}=a_{1}, \phi_{2}=a_{2} \mid \phi_{3}=a_{3}, \phi_{4}=a_{4}\right) P\left(\phi_{3}=a_{3}, \phi_{4}=a_{4}\right) \\
& =P\left(\phi_{1}=a_{1} \mid \phi_{3}=a_{3}\right) P\left(\phi_{2}=a_{2} \mid \phi_{4}=a_{4}\right) P\left(\phi_{3}=a_{3}\right) P\left(\phi_{4}=a_{4}\right) \\
& =P\left(\phi_{1}=a_{1}, \phi_{3}=a_{3}\right) P\left(\phi_{2}=a_{2}, \phi_{4}=a_{4}\right) .
\end{aligned}
$$

Hence, the pairs $\left(\phi_{1}, \phi_{3}\right)$ and $\left(\phi_{2}, \phi_{4}\right)$ are independent.

The following lemma is useful for applying the exact law of large numbers in Corollary 1 to Markov chains.

Lemma 6 Assume that the dynamical system $\mathbb{D}$ is Markov conditionally independent in types. Then, the Markov chains $\left\{\alpha_{i}^{n}\right\}_{n=0}^{\infty}, i \in I$, are essentially pairwise independent. In addition, the processes $h^{n}$ and $g^{n}$ are also essentially pairwise independent for each $n \geq 1$.

Proof. Let $E$ be the set of all $(i, j) \in I \times I$ such that equations (12), (13) and (14) hold for all $n \geq 1$. Then, by grouping countably many null sets together, we obtain that for $\lambda$-almost all $i \in I, \lambda$-almost all $j \in I,(i, j) \in E$, i.e., for $\lambda$-almost all $i \in I, \lambda\left(E_{i}\right)=\lambda(\{j \in I:(i, j) \in$ $E\})=1$. 
We can use induction to prove that for any fixed $(i, j) \in E,\left(\alpha_{i}^{0}, \ldots, \alpha_{i}^{n}\right)$ and $\left(\alpha_{j}^{0}, \ldots, \alpha_{j}^{n}\right)$ are independent, so are the pairs $h_{i}^{n}$ and $h_{j}^{n}$, and $g_{i}^{n}$ and $g_{j}^{n}$. This is obvious for $n=0$. Suppose that it is true for the case $n-1$, i.e., $\left(\alpha_{i}^{0}, \ldots, \alpha_{i}^{n-1}\right)$ and $\left(\alpha_{j}^{0}, \ldots, \alpha_{j}^{n-1}\right)$ are independent, so are the pairs $h_{i}^{n-1}$ and $h_{j}^{n-1}$, and $g_{i}^{n-1}$ and $g_{j}^{n-1}$. Then, the Markov conditional independence condition and Lemma 5 imply that $\left(\alpha_{i}^{0}, \ldots, \alpha_{i}^{n-1}, h_{i}^{n}\right)$ and $\left(\alpha_{j}^{0}, \ldots, \alpha_{j}^{n-1}, h_{j}^{n}\right)$ are independent, so are the pairs $\left(\alpha_{i}^{0}, \ldots, \alpha_{i}^{n-1}, h_{i}^{n}, g_{i}^{n}\right)$ and $\left(\alpha_{j}^{0}, \ldots, \alpha_{j}^{n-1}, h_{j}^{n}, g_{j}^{n}\right)$, and $\left(\alpha_{i}^{0}, \ldots, \alpha_{i}^{n-1}, h_{i}^{n}, g_{i}^{n}, \alpha_{i}^{n}\right)$ and $\left(\alpha_{j}^{0}, \ldots, \alpha_{j}^{n-1}, h_{j}^{n}, g_{j}^{n}, \alpha_{j}^{n}\right)$. Hence, the random vectors $\left(\alpha_{i}^{0}, \ldots, \alpha_{i}^{n}\right)$ and $\left(\alpha_{j}^{0}, \ldots, \alpha_{j}^{n}\right)$ are independent for all $n \geq 0$, which means that the Markov chains $\left\{\alpha_{i}^{n}\right\}_{n=0}^{\infty}$ and $\left\{\alpha_{j}^{n}\right\}_{n=0}^{\infty}$ are independent. It is also clear that for each $n \geq 1$, the random variables $h_{i}^{n}$ and $h_{j}^{n}$ are independent, so are $g_{i}^{n}$ and $g_{j}^{n}$. The desired result follows.

Proof of Theorem 3: (1), (2) and (3) of the theorem are shown in Lemmas 2, 3, and 6 respectively.

By the exact law of large numbers in Corollary 1 , we know that for $P$-almost all $\omega \in \Omega$, $\left(\alpha_{\omega}^{0}, \ldots, \alpha_{\omega}^{n}\right)$ and $\left(\alpha^{0}, \ldots, \alpha^{n}\right)$ (viewed as random vectors) have the same distribution for all $n \geq 1$. Since, as noted in Lemma $4,\left\{\alpha^{n}\right\}_{n=0}^{\infty}$ is a Markov chain with transition matrix $z^{n}$ at time $n-1$, so is $\left\{\alpha_{\omega}^{n}\right\}_{n=0}^{\infty}$ for $P$-almost all $\omega \in \Omega$. Thus (4) is shown.

Since the processes $h^{n}$ and $g^{n}$ are essentially pairwise independent as shown in Lemma 6 , the exact law of large numbers in Lemma 1 implies that at time period $n$, for $P$-almost all $\omega \in \Omega$, the realized cross-sectional distribution after the random mutation, $p^{n}(\omega)=\lambda\left(h_{\omega}^{n}\right)^{-1}$ is the expected cross-sectional distribution $\bar{p}^{n-1 / 2}$, and the realized cross-sectional distribution at the end of period $n, p^{n}(\omega)=\lambda\left(\alpha_{\omega}^{n}\right)^{-1}$ is the expected cross-sectional distribution $\bar{p}^{n}$. Thus, (5) is shown.

To prove (6), note that $\Gamma$ is a continuous function from $\Delta$ to itself. Hence, Brower's Fixed Point Theorem implies that $\Gamma$ has a fixed point $p^{*}$. In this case, $\bar{p}^{n}=\Gamma^{n}\left(p^{*}\right)=p^{*}$, $z_{k l}^{n}=z_{k l}^{1}$ for all $n \geq 1$. Hence the Markov chains $\left\{\alpha_{i}^{n}\right\}_{n=0}^{\infty}$ for $\lambda$-almost all $i \in I,\left\{\alpha^{n}\right\}_{n=0}^{\infty}$, $\left\{\alpha_{\omega}^{n}\right\}_{n=0}^{\infty}$ for $P$-almost all $\omega \in \Omega$ are time-homogeneous. 


\section{References}

[1] R. M. Anderson, A nonstandard representation for Brownian motion and Ito integration, Israel Journal of Mathematics 25 (1976), 15-46.

[2] R. M. Anderson, Non-standard analysis with applications to economics, in Handbook of Mathematical Economics IV (W. Hildenbrand and H. Sonnenschein eds.), North-Holland, New York, 1991.

[3] T. F. Bewley, Existence of equilibria in economies with infinitely many commodities, Journal of Economic Theory 4 (1972), 514-540.

[4] K. Binmore and L. Samuelson, Evolutionary drift and equilibrium selection, Review of Economic Studies 66 (1999), 363-393.

[5] R. T. Boylan, Laws of large numbers for dynamical systems with randomly matched individuals, Journal of Economic Theory 57 (1992), 473-504.

[6] K. Burdzy, D. M. Frankel, and A. Pauzner, Fast equilibrium selection by rational players living in a changing world, Econometrica 69 (2001), 163-189.

[7] L. L. Cavalli-Sforza, and W. F. Bodmer, The Genetics of Human Population, Freeman, San Francisco, 1971.

[8] H. Cole and R. Rogerson, Can the Mortenson-Pissarides matching model match the business-cycle facts?, International Economic Review 40 (1999), 933-959.

[9] E. Dekel and S. Scotchmer, On the evolution of attitudes towards risk in winner-take-all games, Journal of Economic Theory $8 \mathbf{7}$ (1999), 125-143.

[10] P. Diamond, A model of price adjustment, Journal of Economic Theory 3 (1971), 156-168.

[11] P. Diamond, Aggregate demand management in search equilibrium, Journal of Political Economy 90 (1982), 881-894.

[12] P. Diamond and J. Yellin, Inventories and money holdings in a search economy, Econometrica 58 (1990), 929-950.

[13] J. L. Doob, Stochastic processes depending on a continuous parameter, Transactions of the American Mathematical Society 42 (1937), 107-140.

[14] J. L. Doob, Stochastic Processes, Wiley, New York, 1953.

[15] D. Duffie, N. Gârleanu, and L. H. Pedersen, Valuation in over-the-counter markets, Graduate School of Business, Stanford University, 2003.

[16] D. Duffie, N. Gârleanu, and L. H. Pedersen, Over-the-counter markets, Graduate School of Business, Stanford University, 2003.

[17] D. Duffie and Y. N. Sun, Existence of independent random matching, Working paper, 2004 (http://www.stanford.edu/ duffie/exist-J.pdf).

[18] M. Feldman and C. Gilles, An expository note on individual risk without aggregate uncertainty, Journal of Economic Theory 35 (1985), 26-32.

[19] C. A. Ferrer, Dynamical systems with a continuum of randomly matched agents, Journal of Economic Theory 86 (1999), 245-267. 
[20] D. Fudenberg and D. Levine, Steady-state learning and Nash equilibrium, Econometrica 61 (1993), 547-573.

[21] D. Gale, Bargaining and competition, Part I: characterization, Econometrica 54 (1986), 785-806.

[22] D. Gale, Bargaining and competition, Part II: existence, Econometrica 54 (1986), 807-818.

[23] I. Gilboa and A. Matsui, A model of random matching, Journal of Mathematical Economics 21 (1992), 185-197.

[24] E. J. Green, Individual-level randomness in a nonatomic population, Working Paper, University of Minnesota, 1994.

[25] E. J. Green and R. Zhou, Dynamic monetary equilibrium in a random matching economy, Econometrica 70 (2002), 929-970.

[26] G. H. Hardy, Mendelian proportions in a mixed population, Science 28 (1908), 49-50.

[27] J. E. Harrington, The social selection of flexible and rigid agents, American Economic Review 88 (1998), 63-82.

[28] M. Hellwig, A model of monetary exchange, Econometric Research Program, Research Memorandum Number 202, Princeton University, 1976.

[29] A. Hosios, On the efficiency of matching and related models of search and unemployment, Review of Economic Studies 57 (1990), 279-298.

[30] K. L. Judd, The law of large numbers with a continuum of iid random variables, Journal of Economic Theory 35 (1985), 19-25.

[31] H. J. Keisler, Hyperfinite model theory, in Logic Colloquium 76 (R. O. Gandy and J. M. E. Hyland eds.), North-Holland, Amsterdam, 1977.

[32] N. Kiyotaki and R. Wright, On money as a medium of exchange, Journal of Political Economy 97 (1989), 927-954.

[33] N. Kiyotaki and R. Wright, A search-theoretic approach to monetary economics, American Economic Review 83 (1993), 63-77.

[34] J. Krainer and S. LeRoy, Equilibrium valuation of illiquid assets, Economic Theory 19 (2002), 223-242.

[35] L. Ljungqvist and T. Sargent, Recursive Macroeconomic Theory, Boston, MIT Press, 2000.

[36] P. A. Loeb, Conversion from nonstandard to standard measure spaces and applications in probability theory, Transactions of the American Mathematical Society 211 (1975), 113-122.

[37] P. A. Loeb and M. Wolff, eds. Nonstandard Analysis for the Working Mathematician, Kluwer Academic Publishers, Dordrecht, 2000.

[38] J. Maynard-Smith, Evolution and the Theory of Games, Cambridge University Press, Cambridge, UK, 1982.

[39] A. McLennan and H. Sonnenschein, Sequential bargaining as a noncooperative foundation for Walrasian equilibrium, Econometrica 59 (1991), 1395-1424.

[40] M. Merz, Heterogeneous job-matches and the cyclical behavior of labor turnover, Journal of Monetary Economics 43 (1999), 91-124. 
[41] D. Mortensen, Property rights and efficiency in mating, racing, and related games, American Economic Review 72 (1982), 968-979.

[42] D. Mortensen and C. Pissarides, Job creation and job destruction in the theory of unemployment, The Review of Economic Studies 61 (1994), 397-415.

[43] R. Rogerson and R. Wright, Search-theoretic models of labor markets, Working Paper, Department of Economics, University of Pennsylvania, 2002.

[44] P. Rupert, M. Schindler, A. Shevchenko, and R. Wright, The search-theoretic approach to monetary economics: a primer, Working Paper, University of Pennsylvania, 2000.

[45] Y. N. Sun, A theory of hyperfinite processes: the complete removal of individual uncertainty via exact LLN, Journal of Mathematical Economics 29 (1998), 419-503.

[46] Y. N. Sun, The almost equivalence of pairwise and mutual independence and the duality with exchangeability, Probability Theory and Related Fields 112 (1998), 425-456.

[47] Y. N. Sun, The complete removal of individual uncertainty: multiple optimal choices and random economies, Economic Theory 14 (1999), 507-544.

[48] Y. N. Sun, Rich product probability spaces, exact law of large numbers and characterization of insurable risks, Working paper, National University of Singapore, August 2003 (http://www.ims.nus.edu.sg/preprints/2003-25.pdf).

[49] A. Trejos and R. Wright, Search, bargaining, money, and prices, Journal of Political Economy 103 (1995), 118-140.

[50] D. Vayanos and T. Wang, Search and endogenous concentration of liquidity in asset markets, Working Paper, MIT, 2002.

[51] P.-O. Weill, Liquidity premia in dynamic bargaining markets, Working Paper, Stern School of Business, New York University, 2003.

[52] A. Wolinsky, Information revelation in a market with pairwise meetings, Econometrica 58 (1990) $1-23$. 